\title{
Three-year increase of gamma-glutamyltransferase level and development of type 2 diabetes in middle-aged men and women: the D.E.S.I.R. cohort
}

\author{
P. André • B. Balkau • C. Born • M. A. Charles • \\ E. Eschwège $\cdot$ D.E.S.I.R. study group
}

Received: 17 May 2006 / Accepted: 7 July 2006 / Published online: 13 September 2006

(C) Springer-Verlag 2006

\begin{abstract}
Aims/hypothesis Among hepatic markers, gamma-glutamyltransferase (GGT) is the main predictor for development of type 2 diabetes, but there are no data to date on changes in GGT and type 2 diabetes incidence.

Methods Data at baseline and at 3 years from the D.E.S.I.R. cohort were used, comprising 2,071 men and 2,130 women without baseline diabetes.

Results Changes in GGT level were correlated with changes in markers of insulin resistance (fasting insulin, homeostasis model assessment of insulin resistance), as well as with the following elements of the metabolic syndrome: central obesity, and increased fasting glucose, triglycerides and blood pressure (systolic and diastolic). The 3-year increase in GGT was associated with incident type 2 diabetes in both sexes, after adjusting for age and baseline GGT. After further adjustment for baseline confounding factors, including alanine-aminotransferase, alcohol intake, obesity and fasting insulin, the odds ratios $(95 \% \mathrm{CI})$ for an association between incident type 2 diabetes and unchanged or increased (as opposed to decreased) GGT levels were $2.54(1.38-4.68)$ in men $(p=0.003)$ and $2.78(1.20-6.42)$ in women $(p=0.02)$. These associations were slightly attenuated after adjusting for the
\end{abstract}

For details on the D.E.S.I.R. Study Group, see Appendix.

P. André $(\bowtie) \cdot$ B. Balkau $\cdot$ M. A. Charles $\cdot$ E. Eschwège INSERM U780-IFR69,

16 avenue Paul Vaillant-Couturier,

94807 Villejuif Cedex, France

e-mail: andre@vjf.inserm.fr

C. Born

Inter-regional Institute for Health (IRSA),

La Riche, France 3-year change in BMI, alcohol consumption and fasting insulin, the odds ratios being 2.49 (1.28-4.86) in men and 2.53 (1.01-6.40) in women. This relationship was not dependent on intra-individual variability.

Conclusions/interpretation An unchanged or increased GGT level over time, even when GGT is in the normal range, is correlated with increasing insulin resistance and is associated with a risk of incident type 2 diabetes in both sexes, independently of baseline GGT, which is itself a diabetes risk factor.

Keywords GGT · Insulin resistance · Sex · Type 2 diabetes

$\begin{array}{ll}\text { Abbreviations } \\ \text { ALT } & \text { alanine-aminotransferase } \\ \text { GGT } & \text { gamma-glutamyltransferase } \\ \text { HOMA } & \begin{array}{l}\text { homeostasis model assessment of insulin } \\ \text { resistance } \\ \text { OR }\end{array} \\ \text { odds ratio }\end{array}$

\section{Introduction}

Since the original published data in British men [1], recent prospective studies [2-7] have confirmed a significant association between gamma-glutamyltransferase (GGT) level and the incidence of type 2 diabetes, in both sexes and in populations from different countries. This association is independent of classical markers of diabetes, e.g. age and BMI, and also of other factors associated with GGT activity such as excessive alcohol consumption and liver diseases [2-7]. Furthermore when GGT was tested along with other hepatic markers such as alanine-aminotransferase (ALT), aspartate-aminotransferase, bilirubin and 
alkaline phosphatase, GGT was the main predictor of type 2 diabetes [5, 7]. It has also been shown in men that GGT was a significant marker of the metabolic syndrome [5]. The few data that have been published on the longitudinal change in GGT showed a strong association between an increase in GGT and an increase in BMI in both sexes, but other factors were associated differently in men and in women [8].

Our aim was to examine the relationship between changes in GGT levels and changes in factors associated with (1) type 2 diabetes (glycaemia, overall and central obesity), (2) GGT levels (age, alcohol consumption, smoking habits), and (3) insulin resistance markers (fasting insulin, homeostasis model assessment [HOMA]). We also studied whether a change in GGT was associated with incident type 2 diabetes, independently of risk markers for diabetes and insulin resistance.

\section{Subjects and methods}

Study population

This analysis used data from the inclusion and the 3year follow-up examinations of the D.E.S.I.R. cohort study (Data from Epidemiological Study on the Insulin Resistance syndrome), a longitudinal study that aims to clarify the natural history of the insulin resistance syndrome [7]. Among the 5,212 volunteers who participated, the 2,071 men and 2,130 women without diabetes at baseline were included in this analysis, as previously described [7].

\section{Statistical analysis}

Relations between the 3-year change in GGT against baseline and 3-year changes in anthropometric and metabolic variables were examined by Spearman's correlation coefficients.

The associations between the risk of incident type 2 diabetes over 3 years and the 3-year variation in GGT (decrease $[<0]$, increase $[\geq 0]$ ) were analysed separately in men and women, using logistic regression analysis. This comparison was equivalent to comparing those in the highest tertile of GGT change with those in the lower two tertiles. Covariates used were: (1) Baseline age (years), GGT (in quartiles), ALT (in quartiles), BMI, smoking habits (never, former, current), physical activity (four levels), alcohol intake (g/day), fasting insulin $(\mu \mathrm{U} / \mathrm{ml})$ and glucose $(\mathrm{mmol} / \mathrm{l})$. Variables not normally distributed were log transformed. (2) Three-year changes in BMI, alcohol intake and fasting insulin.
For each covariate, interactions with the 3-year change in GGT were tested by likelihood ratio tests, comparing models with and without the interaction terms.

As the intra-individual biological variability of GGT is reported to be $12.2 \%$ [9], we repeated the analyses by comparing participants with an increase of GGT $(>+5 \mathrm{U} / \mathrm{l})$ or a stable GGT $(-5$ to $+5 \mathrm{U} / \mathrm{l})$ value with those with a decreased value $(<-5 \mathrm{U} / \mathrm{l})$.

The SAS statistical program, version 8.0, was used in all analyses.

\section{Results}

Over the 3 years, the mean GGT decreased by $3.0 \mathrm{IU} / 1$ in men and $2.2 \mathrm{IU} / 1$ in women. These 3-year GGT changes were highly and negatively correlated with baseline values for GGT and ALT in both sexes, but not with alcohol intake (Table 1).

GGT change was positively correlated with the change in ALT, fasting glucose, central (WHR) or overall obesity (BMI), insulin resistance (evaluated by fasting insulin, HOMA), and each parameter of the metabolic syndrome (except HDL-cholesterol change), as well as with the change in alcohol intake.

Over the 3 years, 89 subjects developed diabetes: the incidence was $2.8 \%$ in men, $1.4 \%$ in women (Table 2). Participants with increased GGT had an increased risk of developing type 2 diabetes compared with those with a decreased GGT level, with odds ratios (OR) of 1.77 (95\% CI: 1.04-3.02) in men and 3.28 (1.57-6.86) in women, after adjusting for age and baseline GGT. Further adjustment for baseline confounders (BMI, ALT, smoking habits, alcohol intake, physical activity, fasting insulin and glucose) slightly modified this association, though differently in men and women, with ORs of $2.54(1.38-4.68)$ in men and $2.78(1.20-6.42)$ in women. None of the tested interactions were significant.

When the 3-year changes in BMI, fasting insulin or alcohol intake were added separately or all together into the latter model, adjusted for all baseline values (Table 2), the association between increased GGT and type 2 diabetes incidence remained significant with an OR of 2.49 (1.284.86) in men and $2.53(1.01-6.40)$ in women. The ORs were similar when fasting insulin was replaced by the HOMA index or BMI by WHR or waist circumference (data not shown). The ORs for risk of developing type 2 diabetes, adjusted for the same covariates, but comparing participants with an increase in GGT $(>+5 \mathrm{U} / \mathrm{l})$ or a stable GGT value $(-5$ to $+5 \mathrm{U} / \mathrm{l})$ with those with a decrease $(<-5 \mathrm{U} / 1)$, were as follows: increased GGT, $3.03(1.47-6.25)$ in men, 2.69 (0.84-8.59) in women; stable GGT, 1.59 (0.74$3.43)$ in men, $1.67(0.54-5.15)$ in women. 
Table 1 Spearman correlation coefficients between the change in GGT levels over 3 years and both baseline characteristics and 3-year changes in covariates

\begin{tabular}{|c|c|c|c|c|}
\hline & \multicolumn{4}{|c|}{ GGT change over 3 years } \\
\hline & $\begin{array}{l}\text { Men } \\
n=2,071\end{array}$ & $p$ value & $\begin{array}{l}\text { Women } \\
n=2,130\end{array}$ & $p$ value \\
\hline \multicolumn{5}{|l|}{ Baseline characteristics } \\
\hline Age (years) & -0.05 & 0.02 & $<0.01$ & 0.97 \\
\hline GGT (IU/l) & -0.28 & 0.0001 & -0.27 & 0.0001 \\
\hline ALT (IU/l) & -0.23 & 0.0001 & -0.14 & 0.0001 \\
\hline $\operatorname{AST}(\mathrm{IU} / \mathrm{l})$ & -0.13 & 0.0001 & -0.02 & 0.21 \\
\hline Bilirubin (mg/ml) & -0.01 & 0.0001 & $<0.01$ & 0.94 \\
\hline Alcohol intake (g/day) & -0.03 & 0.14 & $<-0.01$ & 0.82 \\
\hline BMI $\left(\mathrm{kg} / \mathrm{m}^{2}\right)$ & -0.08 & 0.0003 & 0.02 & 0.30 \\
\hline Waist-hip-ratio & -0.10 & 0.0001 & -0.01 & 0.77 \\
\hline Fasting insulin $(\mu \mathrm{U} / \mathrm{ml})$ & -0.12 & 0.0001 & -0.05 & 0.02 \\
\hline HOMA & -0.13 & 0.0001 & -0.06 & 0.003 \\
\hline Fasting plasma glucose (mmol/l) & -0.11 & 0.0001 & -0.09 & 0.0001 \\
\hline Triglycerides $(\mathrm{mmol} / \mathrm{l})$ & -0.16 & 0.0001 & -0.05 & 0.02 \\
\hline HDL-cholesterol (mmol/1) & 0.04 & 0.07 & -0.03 & 0.08 \\
\hline Systolic blood pressure $(\mathrm{mmHg})$ & -0.07 & 0.0006 & -0.01 & 0.63 \\
\hline Diastolic blood pressure $(\mathrm{mmHg})$ & -0.13 & 0.0001 & -0.03 & 0.12 \\
\hline \multicolumn{5}{|l|}{ Changes over 3 years } \\
\hline $\operatorname{ALT}(\mathrm{IU} / \mathrm{l})$ & 0.43 & 0.0001 & 0.30 & 0.0001 \\
\hline Alcohol intake (g/day) & 0.09 & 0.0001 & 0.06 & 0.004 \\
\hline BMI $\left(\mathrm{kg} / \mathrm{m}^{2}\right)$ & 0.12 & 0.0001 & 0.15 & 0.0001 \\
\hline Waist-hip-ratio & 0.12 & 0.0001 & 0.07 & 0.0008 \\
\hline Fasting insulin $(\mu \mathrm{U} / \mathrm{ml})$ & 0.14 & 0.0001 & 0.12 & 0.0001 \\
\hline HOMA & 0.15 & 0.0001 & 0.14 & 0.0001 \\
\hline Fasting glucose (mmol/l) & 0.11 & 0.0001 & 0.14 & 0.0001 \\
\hline Triglycerides $(\mathrm{mmol} / \mathrm{l})$ & 0.21 & 0.0001 & 0.14 & 0.0001 \\
\hline HDL-cholesterol (mmol/l) & 0.01 & 0.75 & 0.01 & 0.83 \\
\hline Systolic blood pressure (mmHg) & 0.09 & 0.0001 & 0.04 & 0.07 \\
\hline Diastolic blood pressure $(\mathrm{mmHg})$ & 0.13 & 0.0001 & 0.07 & 0.0005 \\
\hline
\end{tabular}

Data, from the D.E.S.I.R. study, are Spearman correlation coefficients

$A L T$, alanine-aminotransferase; $A S T$, aspartate aminotransferase; HOMA, homeostasis model assessment

\section{Discussion}

In this study, an increase in GGT over 3 years was positively associated with the incidence of type 2 diabetes in both sexes. This association was independent of baseline values of confounding factors including GGT, age, BMI, fasting glucose and insulin. Among baseline hepatic markers, GGT was the main risk factor for type 2 diabetes in this population [7] as well as in a population of Japanese men [5]. Our study is the first to show a correlation between the change in GGT and the change in insulin resistance markers (fasting insulin, HOMA). The HOMA index is a well-validated method for measuring insulin resistance and has shown a good correlation ( 0.58 to 0.88$)$ with reference techniques [10] such as the euglycaemic clamp. The change in GGT had a greater association with change in ALT than with the change in insulin resistance markers or obesity (central or overall). However, the risk of type 2 diabetes in connection with changed GGT levels was only slightly modified after adjustment for changes in these parameters, despite the significant correlations observed with changes in each of these parameters.

Our data support the hypothesis that GGT could be a marker of hepatic steatosis or visceral obesity. GGT change was correlated with the change in ALT (the main marker of non-alcoholic steato-hepatitis) and was also correlated with changes in insulin resistance markers and with obesity indices. However, change in GGT was a risk factor for type 2 diabetes, independently of these markers.

The main limitation of our study is that both type 2 diabetes incidence and the change in GGT were assessed over the same 3 -year period. Type 2 diabetes was diagnosed before or at the same time as the 3-year assessment of GGT. The observed association between the incidence of type 2 diabetes and GGT change could also be explained either by the fact that diabetes status caused a GGT increase as 
Table 2 Odds ratios (95\% CIs) of 3-year incident diabetes for a 3-year increase in GGT, adjusted for baseline values and 3-year changes in covariates

\begin{tabular}{|c|c|c|c|}
\hline & \multicolumn{3}{|c|}{ Change in GGT levels } \\
\hline & Decrease & Increase & $p$ value \\
\hline \multicolumn{4}{|l|}{ Men } \\
\hline Incident cases of diabetes/number of subjects & $32 / 1,312$ & $27 / 759$ & \\
\hline Model A & 1 & $1.77(1.04-3.02)$ & 0.04 \\
\hline Model B & 1 & $2.54(1.38-4.68)$ & 0.003 \\
\hline Model B+BMI change & 1 & $2.44(1.31-4.54)$ & 0.005 \\
\hline Model $\mathrm{B}+$ alcohol intake change & 1 & $2.57(1.39-4.75)$ & 0.003 \\
\hline Model $\mathrm{B}+$ fasting insulin change & 1 & $2.48(1.28-4.81)$ & 0.007 \\
\hline Model B+BMI, alcohol, insulin change & 1 & $2.49(1.28-4.86)$ & 0.008 \\
\hline \multicolumn{4}{|l|}{ Women } \\
\hline Incident cases of diabetes/number of subjects & $13 / 1,487$ & $17 / 643$ & \\
\hline Model A & 1 & $3.28(1.57-6.86)$ & 0.002 \\
\hline Model B & 1 & $2.78(1.20-6.42)$ & 0.02 \\
\hline Model B+BMI change & 1 & $2.42(1.01-5.84)$ & 0.05 \\
\hline Model B+alcohol intake change & 1 & $2.87(1.24-6.67)$ & 0.02 \\
\hline Model $\mathrm{B}+$ fasting insulin change & 1 & $2.82(1.17-6.76)$ & 0.03 \\
\hline Model B+BMI, alcohol, insulin change & 1 & $2.53(1.01-6.40)$ & 0.05 \\
\hline
\end{tabular}

Data are from the D.E.S.I.R. study

Model A: adjusted for age and baseline GGT level

Model B: model A with additional adjustment for baseline alcohol intake, physical activity, smoking habits, alanine-aminotransferase, BMI, fasting insulin and fasting glucose

shown in previous cross-sectional studies [5] where diabetic patients had a higher GGT than non-diabetic patients, or by the fact that the increase in GGT preceded or predicted the onset of diabetes. However, the GGT change was significantly associated with the change in insulin resistance in the whole population and not only in diabetic subjects. As insulin is predictive of diabetes incidence, this association with the change in insulin resistance at least partially supports the hypothesis that the change in GGT preceded diabetes onset,.

This study has two other limitations in connection with the selection of the participants. Firstly, they were volunteers and certainly not representative of the general population, with an under-representation of people with chronic diseases [7]. Secondly, 13\% of the participants were not followed for the 3 years - and they had higher baseline GGT and ALT levels, but similar fasting glucose levels [7]. Nevertheless this selection bias would have a limited impact on the association that clearly exists in this population.

The intra-individual variability of the GGT measurement could also have resulted in a classification bias. Limited changes in GGT over time could be more related to this variability than to a real GGT change. Nevertheless the ORs for developing diabetes, when comparing participants with a GGT increase that was greater than intra-individual variability with patients who had a GGT decrease that was larger than the intra-individual variability, were similar and the association was also significant. Thus the intraindividual variability, including measurement error, cannot explain the reported association between changed GGT levels and type 2 diabetes incidence.

These results, observed in middle-aged adults, support the hypothesis that an increase in GGT over time, even within the normal range, is associated with a change of insulin resistance markers, and with a higher incidence of type 2 diabetes in both sexes, independently of baseline GGT. This association was independent of known confounding factors, of GGT and of the classical diabetes risk markers. GGT is a universally standardised and available measurement that could serve as a clinical marker of the insulin resistance state and its changes.

Acknowledgements This work was supported by cooperative contracts between the INSERM and CNAMTS and Novartis Pharma, by INSERM Public Health Networks and Health Determinants contracts, and by the Vascular Diabetes Risk Association, the French Cardiology Federation, the French Foundation, ALFEDIAM, and ONIVINS. Support was also provided by Ardix Medical, Bayer Diagnostics, Becton Dickinson, Cardionics, Merck Santé, Novo Nordisk, Pierre Fabre and Topcon, who also contributed to the funding of this study.

Duality of interest The authors confirm that they have no duality of interest 


\section{Appendix}

The D.E.S.I.R. Study Group

INSERM U780: B. Balkau, P. Ducimetière, E. Eschwège. INSERM U367: F. Alhenc-Gelas. CHU d'Angers: Y. Gallois, A. Girault. CHU Bichat, INSERM U695: F. Fumeron, M. Marre. Health Examination Centres: Alençon, Angers, Blois, Caen, Chartres, Châteauroux, Cholet, Le Mans, Orléans, Tours. INSTITUT DE RECHERCHE EN MEDECINE GENERALE (IRMG): J. Cogneau, General Practitioners of the area. INSTITUT INTER REGIONAL POUR LA SANTE (IRSA): C. Born, E. Cacès, M. Cailleau, J. G. Moreau, F. Rakotozafy, J. Tichet, S. Vol.

\section{References}

1. Perry IJ, Wannamethee SG, Shaper AG (1998) Prospective study of serum gamma-glutamyltransferase and risk of NIDDM. Diabetes Care 21:732-737

2. Lee DH, Ha MH, Kim JH et al (2003) Gamma-glutamyltransferase and diabetes - a 4-year follow-up study. Diabetologia 46:359-364
3. Nakanishi N, Nishina K, Li W, Sato M, Suzuki K, Tatara K (2003) Serum gamma-glutamyltransferase and development of impaired fasting glucose or type 2 diabetes in middle-aged Japanese men. J Intern Med 254:287-295

4. Lee DH, Jacobs DR Jr, Gross M et al (2003) Gammaglutamyltransferase is a predictor of incident diabetes and hypertension: the Coronary Artery Risk Development in Young Adults (CARDIA) Study. Clin Chem 49:1358-1366

5. Nakanishi N, Suzuki K, Tatara K (2004) Serum gamma-glutamyltransferase and risk of metabolic syndrome and type 2 diabetes in middle-aged Japanese men. Diabetes Care 6:1427-1432

6. Sattar N, Scherbakova O, Ford I et al (2004) Elevated alanineaminotransferase predicts new-onset type 2 diabetes independently of classical risk factors, metabolic syndrome, and C-reactive protein in the west of Scotland coronary prevention study. Diabetes 53:2855-2860

7. Andre P, Balkau B, Born C et al (2005) Hepatic markers and development of Type 2 Diabetes, in middle aged men and women: a three-year follow-up study. Diabetes Metab 31:542-550

8. Nilssen O, Forde OH (1994) Seven-year longitudinal population study of change in gamma-glutamyltransferase: the Tromso Study. Am J Epidemiol 139:787-792

9. Sebastian-Gambaro MA, Liron-Hernandez FJ, Fuentes-Arderiu X (1997) Intra- and inter-individual biological variability data bank. Eur J Clin Chem Biochem 35:845-852

10. Wallace TM, Levy JC, Matthews DR (2004) Use and abuse of HOMA modelling. Diabetes Care 27:1487-1495 